\title{
Deformation of compound shells under action of internal shock wave loading
}

\author{
Marina Chernobryvko ${ }^{1}$, Leopold Kruszka ${ }^{2}$, a , and Konstantin Avramov ${ }^{1}$ \\ ${ }^{1}$ A. N. Podgorny Institute for Mechanical Engineering Problems, National Academy of Sciences of Ukraine, 2/10 Dm. \\ Pozharskoho St., 61046 Kharkiv, Ukraine \\ 2 The General Jaroslaw Dabrowski Military University of Technology, 2 Gen. Sylwester Kaliski St., 00-908 Warsaw, \\ Poland
}

\begin{abstract}
The compound shells under the action of internal shock wave loading are considered. The compound shell consists of a thin cylindrical shell and two thin parabolic shells at the edges. The boundary conditions in the shells joints satisfy the equality of displacements. The internal shock wave loading is modelled as the surplus pressure surface. This pressure is a function of the shell coordinates and time. The strain rate deformation of compound shell takes place in both the elastic and in plastic stages. In the elastic stage the equations of the structure motions are obtained by the assumed-modes method, which uses the kinetic and potential energies of the cylindrical and two parabolic shells. The dynamic behaviour of compound shells is treated. In local plastic zones the 3-D thermo-elastic-plastic model is used. The deformations are described by nonlinear model. The stress tensor elements are determined using dynamic deformation theory. The deformation properties of materials are influenced by the strain rate behaviour, the influence of temperature parameters, and the elastic-plastic properties of materials. The dynamic yield point of materials and Pisarenko-Lebedev's criterion of destruction are used. The modified adaptive finite differences method of numerical analysis is suggested for those simulations. The accuracy of the numerical simulation is verified on each temporal step of calculation and in the case of large deformation gradients.
\end{abstract}

\section{Introduction}

The compound shells are widely used in rocket production and aircraft construction. The compound shell consists of a cylindrical shell and two parabolic shells at the edges. These shells are by the action of internal shock wave loading. The interaction of the thin-walled structures with shock wave can lead to the self-sustained vibrations with large amplitudes and large deformations with destruction.

In spite of wide use of parabolic shells, few efforts were made to analyze dynamics of such structures. Free vibrations of parabolic shells of revolution are analyzed in [1]. The first order shear deformation theory is used to analyze these shells. The discretization of the system by means of the differential quadrature technique leads to the eigenvalue problem. Basing on the firstorder shear deformation theory, the paper [2] focuses on the dynamic behaviour of moderately thick functionally graded parabolic panels and shells of revolution. The generalized differential quadrature method has been used to discretize the system equations.

The article [3] presents the results of an experimental and numerical investigation into the response of partially confined right-circular stainless steel cylinders to air-blast loading. The blast loading was generated by detonating spheres of plastic explosive at two axial positions along the centre line of the cylinders.

\footnotetext{
${ }^{a}$ Corresponding author: 1 kruszka@wat.edu.pl
}

The calculation of material properties following from change of deformation speed, temperature parameters, and elastic-plastic behaviour of materials are analyzed in [4]. Rate-independent finite elastic-plastic equations with thermo-coupled effects are proposed to bypass the yield condition and loading-unloading conditions in [5].

In paper [6] a thermo-elastic-plastic model for the fiber-metal laminated (FML) beams is established. The elastic-plastic behaviour of the FML beam is modelled by a macromechanical plasticity theory, where the composite layers in the FML are assumed to be linearly elastic and the aluminium layers are taken as elastic-plastic. Additionally, the thermal stresses in the FML beam are studied by introducing heat conduction equation. Considering the mismatch in properties of different layers, the interfacial damage is investigated based on cohesive zone model and shear-lag model, and the evolution of the interfacial damage is addressed as well. The incremental thermoelastic-plastic governing equations for the FML beams are solved by finite difference method and iteration method.

In this paper a thermo-elastic-plastic model for the compound shells is investigated. The deformations are described by nonlinear model. The stress tensor elements are determined using dynamic deformation theory. The deformation properties of materials are influenced by the strain rate behaviour, the influence of temperature parameters, and the elastic-plastic properties of materials. The dynamic yield point of materials and PisarenkoLebedev's criterion of destruction are used. 


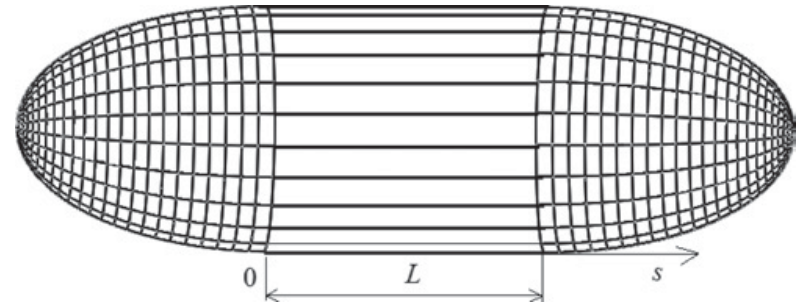

Figure 1. The scheme of the compound shell.

\section{Problem formulation and equations of motions}

We study the construction as the shell consisting of three parts. The compound shell consists of a thin cylindrical shell and two thin parabolic shells at the edges, Fig. 1. The boundary conditions in the shells' joints satisfy the equality of displacements.

The dynamic equations for components of the displacement vector $U_{c}=U(u, w, \beta)$ can be written within the linear Timoshenko theory of shells. For the cylindrical part of the compound shell, the equations of motion are [7]:

$$
\begin{gathered}
\frac{\partial^{2} u}{\partial s^{2}}+\frac{v}{R_{c}} \frac{\partial w}{\partial s}=c_{1} \frac{\partial^{2} u}{\partial t^{2}} \\
\frac{\partial^{2} w}{\partial s^{2}}+\frac{\partial \beta}{\partial s}-b_{1}\left(\frac{w}{R_{c}}+v \frac{\partial u}{\partial s}\right)=c_{2} \frac{\partial^{2} w}{\partial t^{2}}-P_{c}(s, \phi, t) \\
\frac{\partial^{2} \beta}{\partial s^{2}}-b_{2}\left(\frac{\partial w}{\partial s}+\beta\right)=c_{1} \frac{\partial^{2} \beta}{\partial t^{2}}
\end{gathered}
$$

where $s \in[0, L], v-$ Poisson's ratio, $c_{1}=\frac{\rho\left(1-v^{2}\right)}{E}, c_{2}=$ $\frac{\rho}{k G}, \quad b_{1}=\frac{2}{(1-v) k R_{c}}, \quad b_{2}=\frac{6(1-v) k}{h^{2}}, \quad R_{c}-$ radius of the cylindrical middle surface, $h$ - thickness of the cylindrical shell, $k$ - Timoshenko's shear coefficient, $E=E(\dot{\varepsilon}, T)$, $G=G(\dot{\varepsilon}, T)-$ modules of elasticity for tension and compression, which are functions of the strain rate $\dot{\varepsilon}$ and temperature $T, \rho$ - material density, $P_{c}(s, \varphi, t)$ - internal shock wave loading acting on the cylindrical part.

It is necessary to define loading acting on the cylindrical part of the shell. Generally, these fields are determined for each type of explosion in an experimental way. It is possible to take a general form of the loading for numerical experiment:

$$
P_{c}(s, \varphi, t)=Q_{\max }(s, \varphi) e^{-\frac{t-t_{0}(s, \varphi)}{\theta(s, \varphi)}} F_{1}(s, \varphi, t),
$$

where $Q_{\max }(s, \varphi)$ - the characteristic pressure wave amplitude, $\theta(s, \varphi)$ - speed of the pressure wave attenuation, $t_{0}(s, \varphi)$ - detention period of the load act, $F_{1}(s, \varphi, t)$ - distribution function of pressure along cylindrical shell.

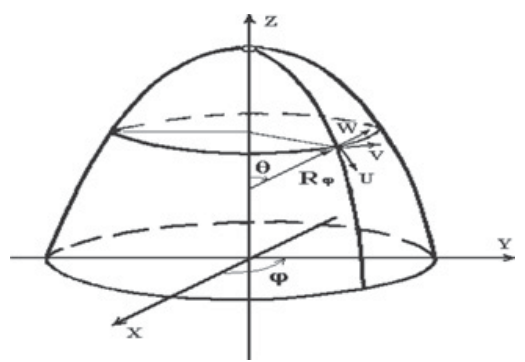

Figure 2. The scheme of the parabolic parts of compound shell.

The displacements $u(s, \varphi, t), w(s, \varphi, t), \beta(s, \varphi, t)$ are expanded using the eigenmodes of free vibrations as:

$$
\begin{gathered}
u(s, \varphi, t)=\sum_{n=1}^{N_{u}} q_{n}^{(u)}(t) U_{n}(s, \varphi) ; \\
w(s, \varphi, t)=\sum_{n=1}^{N_{w}} q_{n}^{(w)}(t) W_{n}(s, \varphi), \\
v(s, \varphi, t)=\sum_{n=1}^{N_{B}} q_{n}^{(B)}(t) B_{n}(s, \varphi) ;
\end{gathered}
$$

where $\quad q^{(u)}=\left[q_{1}^{(u)}, \ldots, q_{N_{u}}^{(u)}\right] ; \quad q^{(w)}=\left[q_{1}^{(w)}, \ldots, q_{N_{w}}^{(w)}\right]$; $q^{(B)}=\left[q_{1}^{(B)}, \ldots, q_{N_{B}}^{(B)}\right]$; are vectors of the generalized coordinates; $U_{n}(s, \varphi), W_{n}(s, \varphi), B_{n}(s, \varphi)$ are eigenmodes of free vibrations.

The scheme of the parabolic parts of compound shell is shown in Fig. 2.

For the parabolic part of the compound shell the potential energy for components of the displacement vector $u(\theta, \varphi, t), v(\theta, \varphi, t), w(\theta, \varphi, t)$ is [7]:

$$
\begin{aligned}
& \Pi=H_{1} \times \int_{0}^{2 \pi} \int_{\theta_{1}}^{\theta_{2}} \\
& \times\left[\left(E_{1}+E_{2}\right)^{2}-2(1-v)\left(E_{1} E_{2}-\frac{1}{4} \Omega_{1}^{2}\right)\right] A_{1} d \theta d \varphi \\
& +H_{2} \int_{0}^{2 \pi} \int_{\theta_{1}}^{\theta_{2}}\left[\left\{\left(K_{1}+K_{2}\right)^{2}-2(1-v)\left(K_{1} K_{2}-\Omega_{2}^{2}\right)\right\}\right. \\
& \times A_{1}+2\left\{\left(E_{1} K_{1}+E_{2} K_{2}\right)+v\left(E_{1} K_{2}+E_{2} K_{1}\right)\right. \\
& \left.\left.+(1-v) \Omega_{1}^{2} \Omega_{2}^{2}\right\} A B_{1}\right] d \theta d \varphi
\end{aligned}
$$

where $R_{0}$ - curvature radius at the shell critical point $\theta=0, v$ - Poisson's ratio, $h-$ the shell thickness; $\rho-$ density of the shell material, $H_{1}=\frac{E h}{2\left(1-v^{2}\right)}, H_{2}=\frac{h^{2}}{12} H_{1}$, 


$$
\begin{aligned}
& A_{1}=R_{0}^{2} \frac{\sin \theta}{\cos ^{4} \theta}, A B_{1}=R_{0} \frac{\sin \theta\left(1+\cos ^{2} \theta\right)}{\cos ^{3} \theta}, \\
& E_{1}= \frac{1}{R_{0}}\left(\frac{\partial u}{\partial \theta}+w\right) \cos ^{3} \theta, \\
& K_{1}= \frac{1}{R_{0}^{2}}\left[\left(\frac{\partial u}{\partial \theta}+\frac{\partial^{2} w}{\partial \theta^{2}}\right) \cos ^{6} \theta\right. \\
&\left.-\left(\frac{\partial w}{\partial \theta}+3 u\right) \cos ^{5} \theta \sin \theta\right], \\
& E_{2}= \frac{1}{R_{0}}\left(\frac{\partial v}{\partial \varphi}+u \cdot \cos \theta+w \cdot \sin \theta\right) \operatorname{ctg} \theta, \\
& K_{2}= \frac{1}{R_{0}^{2}}\left[\frac{\partial v}{\partial \varphi} \operatorname{ctg} \theta \cos \theta-\frac{\partial^{2} w}{\partial \varphi^{2}} \operatorname{ctg}{ }^{2} \theta\right. \\
&\left.+\left(\frac{\partial w}{\partial \theta}-u\right) \operatorname{ctg} \theta \cos ^{4} \theta\right], \\
& \Omega_{1}= \frac{1}{R_{0}}\left(\frac{\partial v}{\partial \theta} \cdot \cos ^{3} \theta-v \cdot \cos ^{2} \theta \sin \theta\right. \\
&\left.+w \cdot \frac{\cos ^{4} \theta}{\sin \theta}+\frac{\partial u}{\partial \varphi} \operatorname{ctg}^{2} \theta\right), \\
& \Omega_{2}= \frac{1}{R_{0}^{2}}\left[\frac{\partial w}{\partial \varphi} \frac{\cos ^{3} \theta}{\sin \theta}-\frac{\cos ^{4} \theta}{\sin \theta}\right. \\
&\left.\times\left(\frac{\partial^{2} w}{\partial \theta \partial \varphi}-\frac{\partial u}{\partial \varphi}-\frac{\partial v}{\partial \theta}+v\right) \frac{\cos ^{4} \theta}{\sin \theta}\right] .
\end{aligned}
$$

The shell kinetic energy takes the following form:

$\mathrm{T}=\frac{\rho h R_{0}^{2}}{2} \int_{0}^{2 \pi} \int_{\theta_{1}}^{\theta_{2}}\left(\left(\frac{\partial u}{\partial t}\right)^{2}+\left(\frac{\partial v}{\partial t}\right)^{2}+\left(\frac{\partial w}{\partial t}\right)^{2}\right) B_{2} d \theta d \varphi$,

where $B_{2}=\frac{\sin \theta}{\cos ^{4} \theta}$.

The virtual work of the internal shock wave loading acting on the parabolic part takes the following form:

$$
\delta \mathrm{A}=R_{0}^{2} \int_{0}^{2 \pi} \int_{\theta_{1}}^{\theta_{2}} P_{p r}(\theta, \varphi, t) \delta w \frac{\sin \theta}{\cos ^{4} \theta} d \theta d \varphi,
$$

where $P_{p r}(\theta, \varphi, t)$-the pressure of the supersonic gas stream acting on the shell; $\delta w$ - virtual displacement of the shell.

In an analogous (2) we can write down $P_{p r}(\theta, \varphi, t)$ for a parabolic shell:

$$
P_{p r}(\theta, \varphi, t)=Q_{\max }(\theta, \varphi) e^{-\frac{t-t_{0}(\theta, \varphi)}{\theta(\theta, \varphi)}} F_{2}(\theta, \varphi, t),
$$

where $F_{2}(\theta, \varphi, t)$ - distribution function of pressure along the parabolic shell.

The displacements $u(\theta, \varphi, t), v(\theta, \varphi, t), w(\theta, \varphi, t)$ are:

$$
\begin{aligned}
& u(\theta, \varphi, t)=\sum_{n=1}^{N_{u}} q_{n}^{(u)}(t) U_{n}(\theta, \varphi) ; \\
& v(\theta, \varphi, t)=\sum_{n=1}^{N_{v}} q_{n}^{(v)}(t) V_{n}(\theta, \varphi) ; \\
& w(\theta, \varphi, t)=\sum_{n=1}^{N_{w}} q_{n}^{(w)}(t) W_{n}(\theta, \varphi),
\end{aligned}
$$

where $q^{(u)}=\left[q_{1}^{(u)}, \ldots, q_{N_{u}}^{(u)}\right] ; q^{(v)}=\left[q_{1}^{(v)}, \ldots, q_{N_{v}}^{(v)}\right] ; q^{(w)}=$ $\left[q_{1}^{(w)}, \ldots, q_{N_{w}}^{(w)}\right]$ are vectors of the generalized coordinates; $U_{n}(\theta, \varphi), V_{n}(\theta, \varphi), W_{n}(\theta, \varphi)$ are eigenmodes of free vibrations [8].

Along the junction of the parabolic shell with the cylinder:

$$
\begin{aligned}
& u(\theta, \varphi, t)=u(s, \varphi, t), \\
& v(\theta, \varphi, t)=v(s, \varphi, t), \\
& w(\theta, \varphi, t)=w(s, \varphi, t) .
\end{aligned}
$$

Let us note that the assumed-modes method determines the displacements for the deformation process.

\section{Thermo-elastic-plastic model}

The strain tensor $\varepsilon_{i j}$ is nonlinear as follows [9]:

$$
\varepsilon_{i j}=\frac{\partial u_{i}}{\partial x_{j}}+\frac{\partial u_{i}}{\partial x_{j}} \frac{\partial u_{j}}{\partial x_{i}} .
$$

The Eq. (10) was used for the parabolic parts.

It should be noted that the system of Eqs. (1) determines the displacements for the deformation process in the cylindrical part. Therefore, the strain-displacement relations can be taken both in the nonlinear as well as in the linearized form. Analysis of the cylindrical shell linearization of the strain-displacement relations does not lead to essential errors. So, the strains are given by:

$$
\varepsilon_{1}=\frac{\partial u}{\partial z}, \quad \varepsilon_{2}=\frac{1}{R_{c}} w, \quad \varepsilon_{3}=\frac{\partial \beta}{\partial z}, \quad \varepsilon_{12}=\frac{\partial w}{\partial z}-\beta,
$$

where $\varepsilon 1$-tensile strain of the medial surface compression in the longitudinal direction, $\varepsilon 2$ - tensile strain of the middle surface in the circumferential direction, $\varepsilon 3$ bending of the surface in the longitudinal direction, $\varepsilon 12$ - shear strain of the middle surface.

In further mathematical modelling of the problem we also take into account that some zones of plastic strain can develop thus forming adiabatic shear bands under detonation loading in the material. Therefore, further solution is reduced by introducing definition of the equivalent strain:

$$
\varepsilon_{e q}=\varepsilon_{e q}\left(\varepsilon_{1}(t), \varepsilon_{2}(t), \varepsilon_{3}(t), \varepsilon_{12}(t)\right)
$$

The stress tensor elements $\sigma_{i i}$ and $\sigma_{i j}$ are determined in a dynamic deformation theory

$$
\begin{aligned}
\sigma_{i i}-\sigma_{0} & =\frac{4 \sqrt{3} \cdot \sigma_{e f f}\left(\varepsilon_{e q}, \dot{\varepsilon}_{e q}, T\right)}{9 \varepsilon_{e q}}\left(\varepsilon_{i i}-\varepsilon_{0}\right), \\
\sigma_{i j} & =\frac{2 \sqrt{3} \cdot \sigma_{e f f}\left(\varepsilon_{e q}, \dot{\varepsilon}_{e q}, T\right)}{3 \varepsilon_{e q}} \varepsilon_{i j}
\end{aligned}
$$

where $\sigma_{0}=\sigma_{i i} / 3$ - mean value of diagonal element of stress tensor, $\varepsilon_{0}=K \sigma_{0}+\alpha T$ - mean value of diagonal element of strain tensor, $\varepsilon_{e q}$ - equivalent strain, $\dot{\varepsilon}_{e q}-$ equivalent strain rate $\sigma_{e f f}$ - effective stress. 


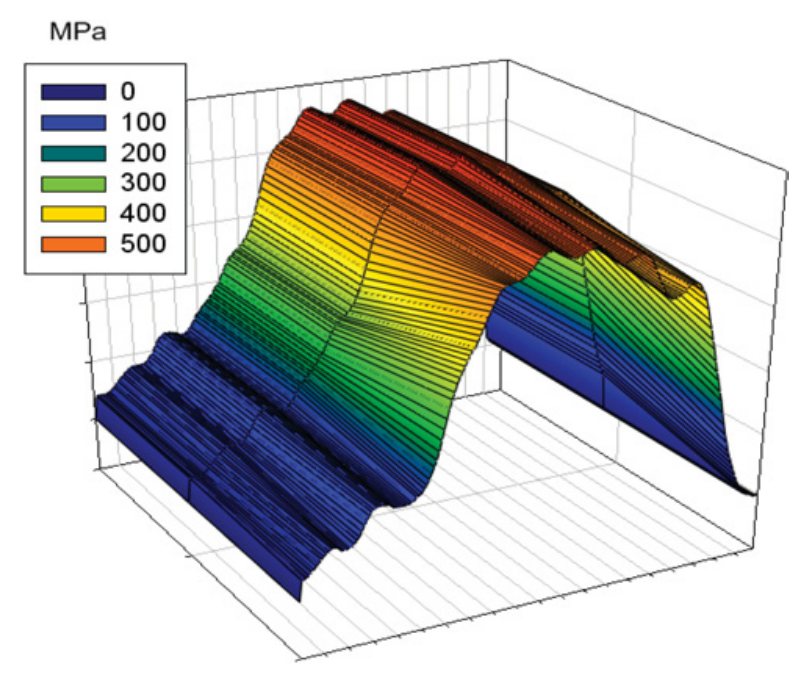

Figure 3. The field of stress obtained along of compound shell.

A multiplier $\sigma_{e f f}\left(\varepsilon_{e q}, \dot{\varepsilon}_{e q}, T\right) / \varepsilon_{e q}$ contains dynamic material properties $\sigma_{e f f}\left(\varepsilon_{e q}, \dot{\varepsilon}_{e q}, T\right)$ :

$$
\begin{aligned}
\sigma_{e f f}= & \sigma_{y}^{s t}\left\{\left[1+\left(\frac{\dot{\varepsilon}_{e q}}{D}\right)^{\frac{1}{n}}\right]-\left(\frac{\varepsilon_{e q}}{\varepsilon_{y}^{s t}}-\left[1+\left(\frac{\dot{\varepsilon}_{e q}}{D}\right)^{\frac{1}{n}}\right]\right)^{m}\right\}_{(14)} \times \\
& \times\left(1-\frac{T-T_{k}}{T_{p}-T_{k}}\right)^{r},
\end{aligned}
$$

where $\sigma_{y}^{s t}$ - the static yield point of materials, $\varepsilon_{y}^{s t}$ - the strain at static yield point, $D, n, m, r$ experimental parameters of dynamic material properties, $T_{k}$ - temperature which determines material properties, $T_{p}$ - melting temperature of material.

The dynamic yield point of materials is as follows:

$$
\sigma_{y}^{d}=\sigma_{y}^{s t}\left[1+\left(\frac{\dot{\varepsilon}_{e q}}{D}\right)^{\frac{1}{n}}\right]
$$

The verification of destruction is conducted with the use of Pisarenko-Lebedev's criterion [10]:

$$
\sigma_{d s t r}=\frac{\sigma_{+}}{\sigma_{-}} \cdot \sigma_{e f f}+\sigma_{11} \cdot\left(1-\frac{\sigma_{+}}{\sigma_{-}}\right),
$$

where $\sigma_{+}-$yield point of materials in tension, $\sigma_{-}-$yield point of materials in compression.

This dynamic problem has a nonlinear character. The finite difference method [9] allows taking an adequate decision on knots of mesh. The choice of small step allows conducting a linear task.

\section{Numerical simulation}

The followings parameters of material were used: $E=1,3 \cdot 10^{11} \mathrm{~Pa} ; E_{1}=10,07 \cdot 10^{8} \mathrm{~Pa} ; \sigma_{y}^{s t}=4,9 \cdot 10^{8} \mathrm{~Pa}$; $\sigma_{d s t r}=6,9 \cdot 10^{8} \mathrm{~Pa} ; \quad \rho=4,5 \cdot 10^{3} \mathrm{~kg} / \mathrm{m}^{3} ; \quad D=4,45$. $10^{3} 1 / \mathrm{s} ; T_{p}=1680^{\circ} \mathrm{C} ; T_{k}=27^{\circ} \mathrm{C} ; \alpha=8,3 \cdot 10^{8} 1 /{ }^{\circ} \mathrm{C}$, $v=0.3 \div 0.5 ; n=4,84 ; m=0,21 ; r=1$.

The results of numerical calculations are presented in Fig. 3.

\section{Summary}

This work presents the strength analysis of the compound shell under the action of internal shock wave loading. This dynamic thermo-elastic-plastic problem is non-linear. Its solution requires to connect mathematical modelling with experimental data of material dynamic characteristics. The main feature of the method is the calculation of influence of the strain hardening and temperature on the dynamic plastic deformation.

This research is partially supported by the grant of National Ukrainian Academy of Sciences in space research for 2012-2016 (contract No. II-67-14).

\section{References}

[1] C. Dahlberg, J. Faleskog, Eur. J. of Mechanics A/Solids 44 (2010), 1-16.

[2] F. Tornabene, E. Viola, Eur. J. of Mechanics A/Solids 28 (2009), 991-1013.

[3] G.S. Langdon, A. Ozinsky, S. Chung Kim, Eur. J. of of Impact Engineering, 73 (2014), 1-14.

[4] M.A. Meyers, Dynamics Behavior of Materials (New York: Wiley, 1994).

[5] L. Kruszka, W.K. Nowacki, J. of Thermal Stresses, 18 (1995), 313-334.

[6] Heng Xiao, Yingli Lia, Yiming Fua, Int. J. of Plasticity, 63 (2014), 211-228.

[7] M. Amabili, Nonlinear Vibrations and Stability of Shells and Plates, 2008, (Cambridge University Press, Cambridge).

[8] M.V. Chernobryvko, K.V. Avramov, V.N. Romanenko, T.J. Batutina, A.M. Tonkonogenko, Int. J. of Theor. and Appl. Mech., 49 (2014), 27-32.

[9] M.V. Chernobryvko, L. Kruszka, Yu.S. Vorobiev Applied Mechanics and Materials, 566, 493-498.

[10] G.S. Pisarenko, A.A. Lebedev, Deformation and strength of materials under complex stress state, 1976, (Naukova Dumka, Kiev). 